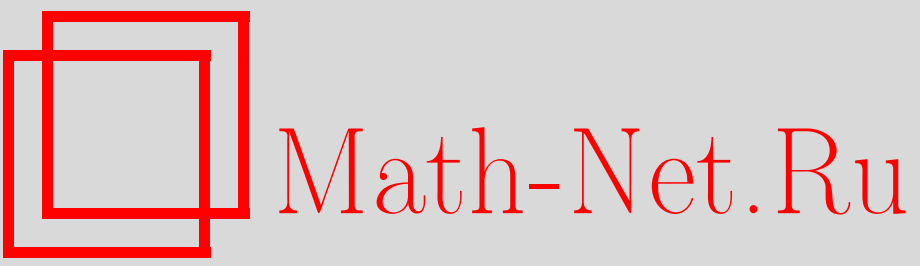

А. И. Жданов, А. А. Иванов, Проекционный регуляризирующий алгоритм для решения некорректных линейных алгебраических систем большой размерности, Вестн. Сам. гос. техн. ун-та. Сер. Физ.-мат. науки, 2010, выпуск 5(), 309-312

DOI: https://doi.org/10.14498/vsgtu827

Использование Общероссийского математического портала Math-Net.Ru подразумевает, что вы прочитали и согласны с пользовательским соглашением

http://www.mathnet.ru/rus/agreement

Параметры загрузки:

IP : 54.162 .127 .20

26 апреля 2023 г., $16: 43: 43$ 
УДК 519.612

\section{ПРОЕКЦИОННЫЙ РЕГУЛЯРИЗИРУЮЩИЙ АЛГОРИТМ ДЛЯ РЕШЕНИЯ НЕКОРРЕКТНЫХ ЛИНЕЙНЫХ АЛГЕБРАИЧЕСКИХ СИСТЕМ БОЛЬШОЙ РАЗМЕРНОСТИ}

\section{А. И. Жданов, А. А. Иванов}

Самарский государственный аэрокосмический университет им. ак. С. П. Королёва (национальный исследовательский университет), факультет информатики (№ 6), 443100, Самара, ул. Молодогвардейская, 151.

E-mails: zhdanov@smr.ru, ssauivanov@gmail.ru

Рассматривается итерационный проекционный алгоритм решения некорректных систем линейных алгебраических уравнений. Данный алгоритм основан на преобразовании регуляризованной нормальной системы уравнений $к$ эквивалентной расширенной регуляризованной нормалъной системе уравнений. Предлагаемый алгоритм позволяет эфбективно решать некорректнье задачи болъшой размерности.

Ключевые слова: итеративный проекционный метод, некорректные задачи, регуляризованные решения.

1. Постановка задачи. Рассмотрим стандартную задачу регуляризации А. Н. Тихонова

$$
\min _{u \in \mathbb{R}^{n}}\left\{\|A u-f\|^{2}+\alpha\|u\|^{2}\right\}
$$

где $A \in \mathbb{R}^{m \times n}, f \in \mathbb{R}^{m}, \alpha>0$ - параметр регуляризации, $\|\cdot\|=\|\cdot\|_{2}$ - евклидова векторная норма.

При большой размерности матрицы $A$ и, возможно, её сильной разреженности часто практически единственно возможным способом решения задачи (1) являются итерационные методы. Однако все итерационные алгоритмы решения задачи (1) основаны на решении уравнений Эйлера (регуляризованных нормальных уравнениях)

$$
\left(A^{\top} A+\alpha I_{n}\right) u=A^{\top} f
$$

где ${ }^{\top}-$ знак транспонирования, $I_{n}-$ единичная матрица порядка $n$.

C учётом того, что число обусловленности задачи (2) равно приблизительно квадрату числа обусловленности исходной задачи (1), многие плохо обусловленные задачи вида (1) решить известными итерационными методами практически невозможно.

В данной работе предполагается, что параметр регуляризации $\alpha$ известен. Например, эффективным способом выбора параметра регуляризации является результат, полученный В.А. Морозовым и С.Ф. Гилязовым [1]. В соответствии с этим подходом параметр регуляризации $\alpha$ выбирается $\alpha=h$, где $h$-величина, характеризующая погрешность задания элементов матрицы $A$.

2. Метод расширенных регуляризованных нормальных уравнений. Как показано

Александр Иванович ЖХанов (д.ф.-м.н., проф.), зав. кафедрой, каф. прикладной математики. Андрей Александрович Иванов, магистрант, каф. прикладной математики. 
в [2], регуляризованную нормальную систему уравнений (2) можно записать в виде

$$
\left(\begin{array}{cc}
\omega I_{m} & A \\
A^{\top} & -\omega I_{n}
\end{array}\right)\left(\begin{array}{l}
y \\
u
\end{array}\right)=\left(\begin{array}{l}
f \\
0
\end{array}\right) \quad \Longleftrightarrow \quad \tilde{A}_{\omega} z=\tilde{f},
$$

где $\omega=\sqrt{\alpha}$.

Матрица $\tilde{A}_{\omega}$ системы (3) при всех $\alpha>0$ невырождена [2] и её единственным решением является вектор $z_{*}=\left(y_{*}^{\top}, u_{*}^{\top}\right)^{\top}$, где $u_{*}=\left(A^{\top} A+\alpha I_{n}\right)^{-1} A^{\top} f, y_{*}=\omega^{-1} r_{*}$, $r_{*}=f-A u_{*}$.

Спектральное число обусловленности регуляризованной нормальной системы $(2)$ составляет величину

$$
\kappa_{2}\left(A^{\top} A+\alpha I_{n}\right)=\frac{\sigma_{\max }^{2}+\alpha}{\sigma_{\min }^{2}+\alpha},
$$

где $\sigma_{\min }$ и $\sigma_{\max }-$ минимальное и максимальное сингулярные числа матрицы $A$ соответственно. При этом спектральное число обусловленности расширенной регуляризованной нормальной системы $(3)$ существенно меньше и равно величине $\kappa_{2}\left(\tilde{A}_{\omega}\right)=$ $=\sqrt{\kappa_{2}\left(A^{\top} A+\alpha I_{n}\right)}$.

3. Проекционный алгоритм. Запишем расширенную систему (3) в виде системы двух уравнений:

$$
\begin{gathered}
\left(\omega I_{m} \vdots A\right) z=f, \\
\left(A^{\top}:-\omega I_{n}\right) z=0 .
\end{gathered}
$$

Для решения системы (4), (5) используем блочный вариант алгоритма Качмажа $[4,5]$ (проекционный алгоритм):

$$
\begin{gathered}
z_{i, 1}=z_{i, 0}-\left(\omega I_{m} \vdots A\right)^{+}\left[\left(\omega I_{m} \vdots A\right) z_{i, 0}-f\right] \\
z_{i, t}=z_{i, t-1}-\beta_{i, t-1}\left(\begin{array}{c}
a_{t-1} \\
-\omega e_{t-1}
\end{array}\right), \\
\beta_{i, t-1}=\frac{\left(a_{t-1}^{\top},-\omega e_{t-1}^{\top}\right) z_{i, t-1}}{\left\|\left(a_{t-1}^{\top},-\omega e_{t-1}^{\top}\right)^{\top}\right\|^{2}}, \quad t=2,3, \ldots, n+1, \\
z_{i+1,0}=z_{i, n+1}, \quad i=1,2, \ldots,
\end{gathered}
$$

где $i$-номер внешних итераций, а $t$-номер внутренних итераций (общее число внутренних итераций в алгоритме $(6),(7)$ равно $n+1),\left(e_{1}, e_{2}, \ldots, e_{n}\right)=I_{n} ; A=$ $=\left(a_{1}, a_{2}, \ldots, a_{n}\right) ;(\cdot)^{+}$- псевдообратная матрица.

Матрица $\tilde{A}_{\omega}$ невырождена при всех $\alpha>0$ [2], следовательно $[4,5], z_{i, n+1} \stackrel{i \rightarrow \infty}{\longrightarrow} z_{*}$ при любом начальном значении вектора $z_{1,0}$.

Если обозначить $u=\left(u^{(1)}, \ldots, u^{(n)}\right)^{\top}$, то учитывая, что вектор $z=\left(y^{\top}, u^{\top}\right)^{\top}$, рекуррентное уравнение (7) можно записать в виде двух рекуррентных уравнений:

$$
\begin{aligned}
y_{i, t} & =y_{i, t-1}-\beta_{i, t-1} a_{t-1}, \quad \beta_{i, t-1}=\frac{a_{t-1}^{\top} y_{i, t-1}-\omega u_{i, t-1}^{(t-1)}}{\left\|a_{t-1}\right\|^{2}+\omega^{2}}, \\
u_{i, t}^{(t-1)} & =u_{i, t-1}^{(t-1)}+\omega \beta_{i, t-1}, \quad t=2,3, \ldots, n+1 .
\end{aligned}
$$

Так как $y=\omega^{-1} r$, где $r=f-A u$, рекуррентные уравнения (9), (10) можно преобразовать к виду

$$
r_{i, t}=r_{i, t-1}-\rho_{i, t-1} a_{t-1}, \quad \rho_{i, t-1}=\frac{a_{t-1}^{\top} r_{i, t-1}-\alpha u_{i, t-1}^{(t-1)}}{\left\|a_{t-1}\right\|^{2}+\alpha},
$$




$$
u_{i, t}^{(t-1)}=u_{i, t-1}^{(t-1)}+\rho_{i, t-1}, \quad t=2,3, \ldots, n+1 .
$$

В [5] отмечалось, что подразделение итерационных шагов $u_{i, t}$ и $r_{i, t}$ на внутренние и внешние в уравнениях (11), (12) является необязательным, и вычислительный процесс можно представить как расчёт «микроитераций» по некоторому одному параметру $k=1,2 \ldots$ Для сходимости таких приближений необходимо только, чтобы в последовательности векторов $a_{1}, a_{2}, \ldots$ участвовали все столбцы матрицы $A$. Более того, будет показано, что при выполнении некоторого дополнительного условия согласования начальных условий $u_{0}=u_{1,0}$ и $r_{0}=r_{1,0}$ использование первого рекуррентного уравнения (6) в итерационном процессе вообще не требуется. Уравнение (4) используется только для согласования начальных значений $u_{0}$ и $r_{0}$.

Пусть $k=1,2 \ldots$ и $j(k)=(k-1) \bmod (n)+1$. Следовательно, $\{j(k)\}_{k=1}^{\infty}$ является периодической последовательностью вида $1,2, \ldots, n, 1,2, \ldots, n, \ldots$ Тогда рекуррентные уравнения (11), (12) можно записать в виде

$$
\begin{aligned}
& r_{k}=r_{k-1}-\rho_{k-1} a_{j(k)}, \\
& u_{k}=u_{k-1}+\rho_{k-1} e_{j(k)},
\end{aligned}
$$

где

$$
\rho_{k-1}=\frac{a_{j(k)}^{\top} r_{k-1}-\alpha e_{j(k)}^{\top} u_{k-1}}{\left\|a_{j(k)}\right\|^{2}+\alpha}, \quad k=1,2, \ldots
$$

В уравнениях (13), (14) индекс $k$ и индексы $i, t$ и $n$ в уравнениях $(11),(12)$ связаны соотношением $k=(i-1) n+t-1$, а $t-1=j(k)$. Очевидно, что тогда $r_{0}=r_{1,0}$ и $u_{0}=u_{1,0}$.

Введем вектор $\theta_{k}=\left(r_{k}^{\top}, u_{k}^{\top}\right)^{\top}$. Тогда рекуррентные уравнения (13), (14) можно записать в виде одного рекуррентного уравнения

$$
\theta_{k}=\theta_{k-1}-\rho_{k-1}\left(\begin{array}{c}
a_{j(k)} \\
-e_{j(k)}
\end{array}\right), \quad \rho_{k-1}=\frac{\left(a_{j(k)}^{\top},-\alpha e_{j(k)}^{\top}\right) \theta_{k-1}}{\left\|a_{j(k)}\right\|^{2}+\alpha}, \quad k=1,2 \ldots,
$$

где $\theta_{0}-$ вектор начальных значений.

ТеОрема. Пусть в рекуррентном уравнении (15) вектор $\theta_{0}=\left(r_{0}^{\top}, u_{0}^{\top}\right)^{\top}$ удовлетворяет условию согласования

$$
r_{0}=f-A u_{0}
$$

Тогда для произвольного начального вектора $u_{0} \quad \theta_{k} \longrightarrow \theta_{*}$ при $k \longrightarrow \infty$, где $\theta_{*}=$ $=\left(r_{*}^{\top}, u_{*}^{\top}\right)^{\top}$.

Доказ ательств о. Докажем по индукции, что из условия согласованности (16) начальных значений следует выполнение условия согласованности

$$
r_{k}=f-A u_{k}
$$

при любых $k \geqslant 0$, где $r_{k}$ и $u_{k}$ вычисляются из рекуррентных уравнений $(13),(14)$.

При $k=1$ из (13), (14) получаем

$$
f-A u_{1}=f-A\left(u_{0}+\rho_{0} e_{1}\right)=\left(f-A u_{0}\right)-\rho_{0} a_{1}=r_{0}-\rho_{0} a_{1}=r_{1} .
$$

Таким образом, для $k=1$ условие согласованности (17) выполняется.

Пусть (17) выполняется для некоторого произвольного $k=\nu>1$. Покажем, что тогда из справедливости (17) для $k=\nu$ следует справедливость (17) при $k=\nu+1$.

Из рекуррентных уравнений (13), (14) непосредственно получаем $f-A u_{j(\nu+1)}=f-A\left(u_{\nu}+\rho_{\nu} e_{j(\nu+1)}\right)=\left(f-A u_{\nu}\right)-\rho_{\nu} a_{j(\nu+1)}=r_{\nu}-\rho_{\nu} a_{j(\nu+1)}=r_{j(\nu+1)}$. 
Из этого следует справедливость (17) для любых $k \in \mathbb{N}$.

Если в рекуррентных уравнениях $(6),(7) y_{1,0}$ и $u_{1,0}$ удовлетворяют условию

$$
\omega y_{1,0}=f-A u_{1,0},
$$

то $z_{1,1}=z_{1,0}$ и, следовательно, векторы $r_{i, t}$ и $u_{i, t}$ из $(11),(12)$ при $k=(i-1) n+t-1$ полностью совпадает с вектором $\theta_{k}$ из уравнения (15). Из справедливости условия (17) для всех $k=1,2 \ldots$ непосредственно получаем, что $z_{i, 1}=z_{i, 0}$ и рекуррентное уравнение (6) при выполнении условия (16) не требуется.

Окончательно из полной эквивалентности рекуррентных уравнений (11), (12) и (15) при выполнении условия (16) следует справедливость теоремы.

Выводы. Проекционный алгоритм (15) эффективен при решении задач регуляризации (4) большой размерности, так как для его реализации на одной итерации требуются лишь две матричные операции: скалярное произведение и операция saxpy.

Работа выполнена при поддержке РФФИ (код проекта № 10-01-00723-а).

\section{БИБЛИОГРАФИЧЕСКИЙ СПИСОК}

1. Морозов В.А. Алгоритмические основы методов решения некорректно поставленных задач // Вычисл. методы и программирование, 2003. - Т. 45. - С. 130-141.

2. Жданов А.И. Об одном численно устойчивом алгоритме решения систем линейных алгебраических уравнений неполного ранга // Вестн. Сам. гос. техн. ун-та. Сер. Физ.мат. науки, 2008. - № 1(16). - C. 149-153.

3. Kaczmarz S. Angenäherte Aufösung von Systemen linearen Gleichunngen// Bull. Acad. Polon. Sci. Lett. A, 1937. - Vol. 35. - P. 355-357.

4. Василъченко Г. П., Светлаков А. А. Проекционный алгоритм решения систем линейных алгебраических уравнений большой размерности // Журн. вычисл. математики и мат. физики, 1980. - Т. 20, № 1. - С. 3-10.

5. Ильин В. П. Об итерационном методе Качмажа и его обобщениях // Сиб. журн. индустр. матем., 2006. - Т. 9, № 3. - С. 39-49.

Поступила в редакцию 02/IX/2010;

в окончательном варианте $-13 / \mathrm{X} / 2010$.

MSC: $65 \mathrm{~F} 10$

\section{PROJECTION REGULARIZATION ALGORITHM FOR SOLVING LINEAR ALGEBRAIC SYSTEM OF LARGE DIMENSION}

\section{A. I. Zhdanov, A. A. Ivanov}

S. P. Korolyov Samara State Aerospace University

(National Research University), Faculty of Computer Science,

151, Molodogvardeyskaya st., Samara, 443100, Russia.

E-mails: zhdanov@smr.ru, ssauivanov@gmail.ru

The iterative projection algorithm for solving ill-posed systems of linear algebraic equations is examined. This algorithm is based on transforming the regularized normal equations to the equivalent augmented regularized normal system of equations. The proposed algorithm can effectively solve ill-posed problems of large dimension.

Key words: iterativ projection method, incorrect problems, regularized solution.

Original article submitted 02/IX/2010; revision submitted $13 / \mathrm{X} / 2010$.

Alexander I. Zhdanov (Dr. Sci. (Phys. \& Math)), Head of Dept., Dept. of Applied Mathematics. Andrey A. Ivanov, Master Student, Dept. of Applied Mathematics. 